\title{
Caracterização, atributos e potencial de mercado do mel de cacau
}

\author{
Characterization, attributes and market potential of cocoa honey \\ Caracterización, atributos y potencial de mercado de lo miel de cacao
}

Recebido: 17/03/2021 | Revisado: 02/04/2021 | Aceito: 06/04/2021 | Publicado: 16/04/2021

\author{
Christiano Pedro Guirlanda \\ ORCID: https://orcid.org/0000-0002-7909-6951 \\ Universidade Federal de Minas Gerais, Brasil \\ E-mail: alimenttos@gmail.com \\ Geisa Gabriela da Silva \\ ORCID: https://orcid.org/0000-0002-5596-8698 \\ Universidade Federal de Minas Gerais, Brasil \\ E-mail: geisambio@gmail.com \\ Jacqueline Aparecida Takahashi \\ ORCID: https://orcid.org/0000-0002-8831-1609 \\ Universidade Federal de Minas Gerais, Brasil \\ E-mail: jat@qui.ufmg.br
}

\begin{abstract}
Resumo
O mel de cacau é um subproduto da produção de cacau, cujo volume vem aumentando com o crescimento da indústria de chocolates e derivados. Este líquido produzido naturalmente durante a pós-colheita do cacau tem sabor agridoce e cor clara, possui compostos bioativos, açúcares, vitamina $\mathrm{C}$ e fibras dietéticas de importância para a indústria de alimentos. Sua aplicação industrial ainda requer estudos aprofundados e desenvolvimento tecnológico, porém, a literatura já aponta pesquisas para desenvolvimento de novos produtos contendo mel de cacau como sorvetes, geleias mistas, vinagres e bebidas alcoólicas. Atualmente o aproveitamento do mel de cacau ocorre de forma tímida, muitas vezes artesanal, limitada às propriedades produtoras de cacau devido a restrições para seu uso industrial e questões como sua conservação, que hoje em dia ocorre basicamente pelo processo de congelamento. Com os atuais esforços para fortalecimento da bioeconomia e de exploração sustentável das cadeias produtivas, há uma grande necessidade de desenvolvimento de produtos a partir de mel de cacau, de forma a evitar seu descarte e agregar renda para os produtores. Esta revisão descreve a obtenção, a composição físico-química, o potencial bioativo e tecnológico do mel
\end{abstract} de cacau, dentro da cadeia de beneficiamento do cacau.

Palavras-chave: Mel de cacau; Suco natural; Valorização de subprodutos; Bioeconomia; Indústria de chocolate.

\begin{abstract}
Cocoa honey is a by-product of cocoa production and its volume has been increasing with the growth of chocolate and derivatives industry. This liquid produced naturally during cocoa post harvesting has bittersweet flavor and light color, presents bioactive compounds, sugars, vitamin $\mathrm{C}$ and dietary fibers, compounds of importance to the food industry. Its industrial application still requires in-depth studies and technological development; however, the literature already points to research for the development of new products containing cocoa honey such as ice cream, mixed jams, vinegars and alcoholic beverages. Currently the use of cocoa honey occurs in a timid, often artisanal way, limited to cocoa-producing properties due to restrictions on its industrial use and issues such as its conservation, which nowadays occurs basically by freezing process. With the need to strengthen the bioeconomy and make a sustainable use of the production chains, there is a great need to develop products from cocoa honey, in order to avoid its disposal and add income to producers. This review describes the obtaining, physical-chemical composition, bioactive and technological potential of cocoa honey, within the cocoa processing chain.
\end{abstract}

Keywords: Cocoa honey; Natural juice; Subproduct valorization; Bioeconomy; Chocolate industry.

\section{Resumen}

El miel de cacao es un subproducto de la producción de cacao, cuyo volumen ha ido aumentando con el crecimiento de la industria del chocolate y los derivados. Este líquido producido naturalmente durante el cacao postharvest tiene sabor agridulce y color claro, compuestos bioactivos, azúcares, vitamina $\mathrm{C}$ y fibras dietéticas de importancia para la industria alimentaria. Su aplicación industrial todavía requiere estudios en profundidad y desarrollo tecnológico, sin embargo, la literatura ya apunta a la investigación para el desarrollo de nuevos productos que contienen miel de cacao como helados, mermeladas mixtas, vinagres y bebidas alcohólicas. Actualmente el uso de miel de cacao se produce de una manera tímida, a menudo artesanal, limitada a las propiedades productoras de cacao debido a las restricciones en su uso industrial y cuestiones como su conservación, que hoy en día se produce básicamente por proceso de congelación. Con la necesidad de fortalecer la bioeconomía y explotar de manera sostenible las cadenas de 
producción, existe una gran necesidad de desarrollar productos a partir de miel de cacao, a fin de evitar su eliminación y agregar ingresos a los productores. Esta revisión describe el logro, la composición físico-química, el potencial bioactivo y tecnológico de lo miel de cacao, dentro de la cadena de procesamiento de cacao.

Palabras clave: Miel de cacao; Jugo natural; Valorización de subproductos; Bioeconomía; Industria del chocolate.

\section{Introdução}

O consumo médio mundial de chocolate é estimado em $0,9 \mathrm{~kg}$ per capita por ano, mas este valor é muito maior em alguns países da Europa, o maior mercado produtor e exportador de chocolate do mundo (CBI, 2020). Dados recentes apontam a Alemanha como a maior consumidora mundial de chocolate, com consumo de $11 \mathrm{~kg}$ per capita em 2018, seguida pela Suíça (9,7 kg) e Estônia (8,8 kg). Desta forma, o consumo médio per capita de chocolate na Europa chegou a 5,0 kg em 2018 (CBI, 2020). O mercado europeu de chocolate foi avaliado em cerca de $€ 53$ bilhões em 2019 e deve apresentar crescimento a uma taxa média anual de cerca de 5\% entre 2020 e 2024. A pandemia do COVID-19 impactou o mercado de forma variada, com queda na demanda de alguns segmentos. Percebem-se, porém, oportunidades de crescimento promissoras, como o mercado de chocolates sazonais e o varejo online, que impulsonaram o mercado de chococlate principalmente a Europa ocidental com uma projeção de valor do mercado europeu de US\$72,04 bilhões para 2024 (Businesswire, 2020).

A região Ásia-Pacífico continua sendo o mercado mais forte para os ingredientes de cacau, cujo crescimento tornou esta região na segunda maior em volume de ingredientes, à frente da América do Norte (Euromonitor, 2016). Para atender a essa demanda de chocolate, foram produzidos no mundo cerca de 4,697 milhões de toneladas nas safras 2019/2020, o que demonstra queda de produção em relação à safra 2018/2019 (1,8\%). Oito países juntos foram responsáveis por 4194 milhões de toneladas desta produção mundial de amêndoas, conforme apresentado na Figura 1.

Figura 1 - Estimativa de produção de cacau no mundo entre 2017 e 2020.

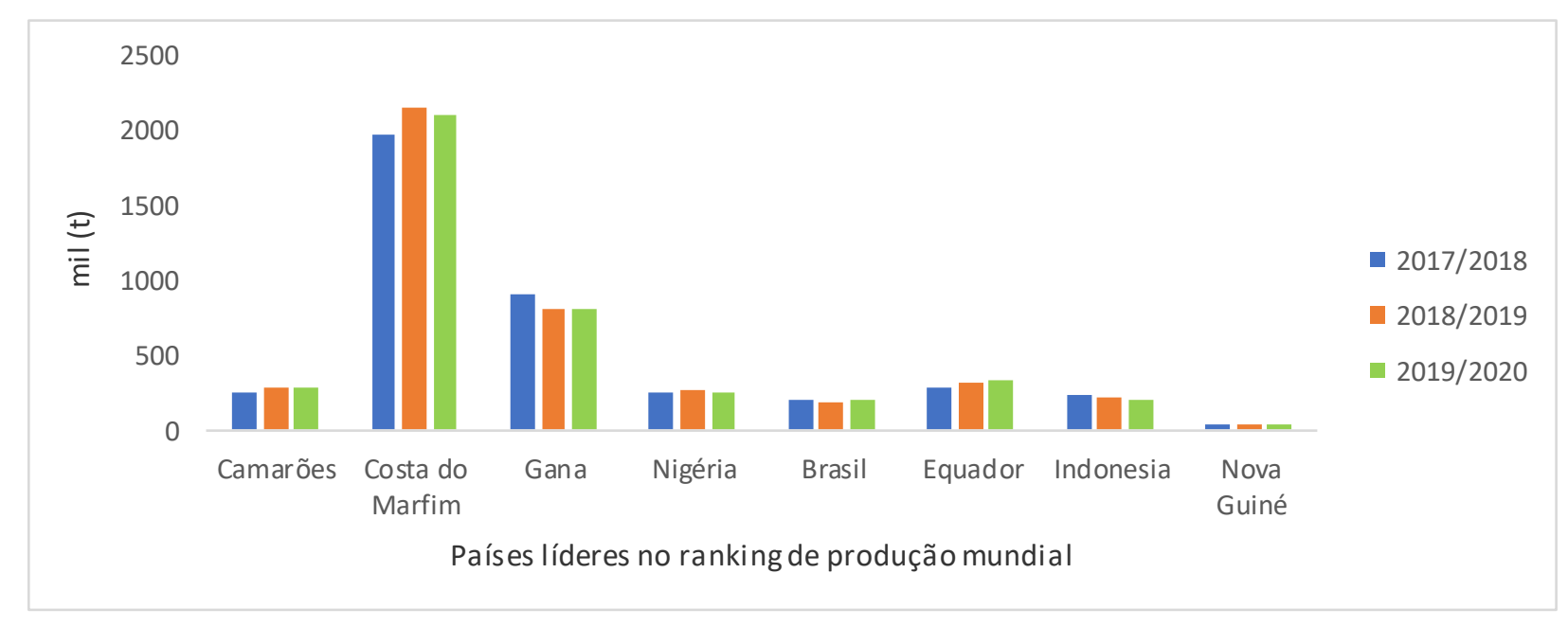

Fonte: ICCO (2020)

Mesmo com tendência crescente de processamento do cacau nas regiões produtoras, mais da metade do cacau no mundo é industrializado em regiões que, embora não produzam cacau, são fabricantes tradicionais de chocolate. As cinco maiores indústrias chocolateiras são Mars (EUA); Ferrero (Itália); Mondelez (EUA); Meiji (Japão) e Nestlé (Suíça) (Brasil, 2018). O Brasil é um raro caso de país com a cadeia produtiva completa, pois é produtor de cacau, possui parque industrial de processamento da amêndoa e é fabricante de chocolate. 
Apesar do impacto na produção nacional causada pela crise econômica devido à pandemia do Corona vírus, foi registrado um crescimento da produção de chocolates em 1,97\% no terceiro trimestre de 2020 em comparação ao mesmo período do ano de 2019, crescimento este impulsionado pelo consumo de tabletes de chocolate que apresentaram alta de $28,4 \%$ (ABICAB 2020). Dados de mercado também mostram que 55,4\% dos brasileiros compram chocolate mensalmente, levando a 9\% de incremento no faturamento da categoria de chocolate, no comparativo com o mesmo período de 2019, atingindo valores estimados em R \$ 5,4 bilhões até setembro (Mercado do cacau, 2020).

A produção de cacau tem peso importante na economia brasileira, apesar de problemas com estiagem, preço baixo e pragas. Na Bahia, o aumento da produtividade por hectare e promoção da verticalização da cadeia produtiva no sul do estado. Também merece destaque o forte crescimento em produtividade da produção de cacau no Estado do Pará, cuja participação no cenário nacional passou de 18\% em 2005, para 53\% em 2018. No ano de 2018, o Pará produziu mais de 116 mil toneladas de cacau, em aproximadamente 180 mil hectares, área plantada que equivale a menos da metade da extensão destinada à colheita na Bahia, que produziu no mesmo período pouco mais de 122 mil toneladas (CEPLAC, 2019).

O cacau sempre foi associado com exploração econômica de suas amêndoas para produção de chocolate. A indústria de transformação de cacau gera uma quantidade significativa de resíduos principalmente na etapa de pós-colheita, dos quais os principais são a casca do cacau, a polpa e o mel de cacau (Ruesgás-Ramón et al., 2020).

\section{Metodologia}

Foi realizada pesquisa documental através de revisão da bibliografia com vista na obtenção de dados da cadeia produtiva, potencial de mercado, conservação e caracterização físico-química do mel de cacau. A abordagem qualitativa dos métodos foi utilizada na medida em que foi importante a interpretação de dados por parte do pesquisador, tendo sido adicionadas opiniões dos autores sobre o fenômeno em estudo (Pereira et al. 2018).

\section{O Processamento do Fruto de Cacau}

A amêndoa do cacau representa a principal matéria-prima para a produção do chocolate. Para obtenção das amêndoas, os frutos maduros do cacau são coletados no campo, suas cascas fibrosas são quebradas e as amêndoas revestidas com uma polpa clara e mucilaginosa são removidas do interior do fruto. No momento em que amêndoas e polpa são expostas ao ar, inicia-se um processo de fermentação espontânea. A massa de amêndoas obtida é inserida em cochos de madeira para a continuação do processo fermentativo, que é necessário para remover a polpa. A fermentação também influencia na formação das características sensoriais de cor e sabor do chocolate. Os processos fermentativos que ocorrem na pós colheita do cacau tem duas fases distintas, uma aeróbica e outra anaeróbica, em um processo de fermentação complexo com participação de leveduras e bactérias (Lima et al., 2011). Após a fermentação, os grãos passam por secagem, torrefação e aquecimento, sendo posteriormente triturados. Nesta etapa, a casca dos grãos é retirada e, a partir desta etapa, segue-se a fabricação de produtos como "nibs", manteiga e amêndoas de cacau, que seguem para a produção de chocolate e outros produtos como achocolatado. Durante a fermentação, um suco transparente é drenado da massa de cacau. Este suco possui características químicas e sensoriais similares à polpa original é chamado regionalmente de mel de cacau (Santos et al., 2014), também citado na literatura como "suor" de cacau (Balladares et al., 2016) e exsudado de cacau (Garcia \& Moreta, 2013). Na literatura internacional é referido como cocoa honey e cocoa sweatings. A Figura 2 apresenta as etapas do processamento do cacau, incluindo a etapa de obtenção de mel de cacau. O mel de cacau geralmente é subaproveitado em grande parte das propriedades produtoras de amêndoas de cacau (Ruesgás-Ramón et al., 2020). O mel de cacau também pode ser obtido utilizando prensas mecânicas construídas em madeira de forma artesanal ou com materiais como o aço inox que garantem a qualidade do produto 
final para fins industriais (Freire et al.,1990). Outro método de extração da polpa utiliza despolpadeira combinada com pulverização de enzimas pecnolíticas na concentração de $0,2 \%$, ocorrendo diminuição da viscosidade foi reduzida e aumento do rendimento devido à quebra da pectina, além de aumento da concentração dos açúcares (Freire et al., 1990).

Figura 2 - Processamento do cacau para obtenção das amêndoas secas e do subproduto mel de cacau.

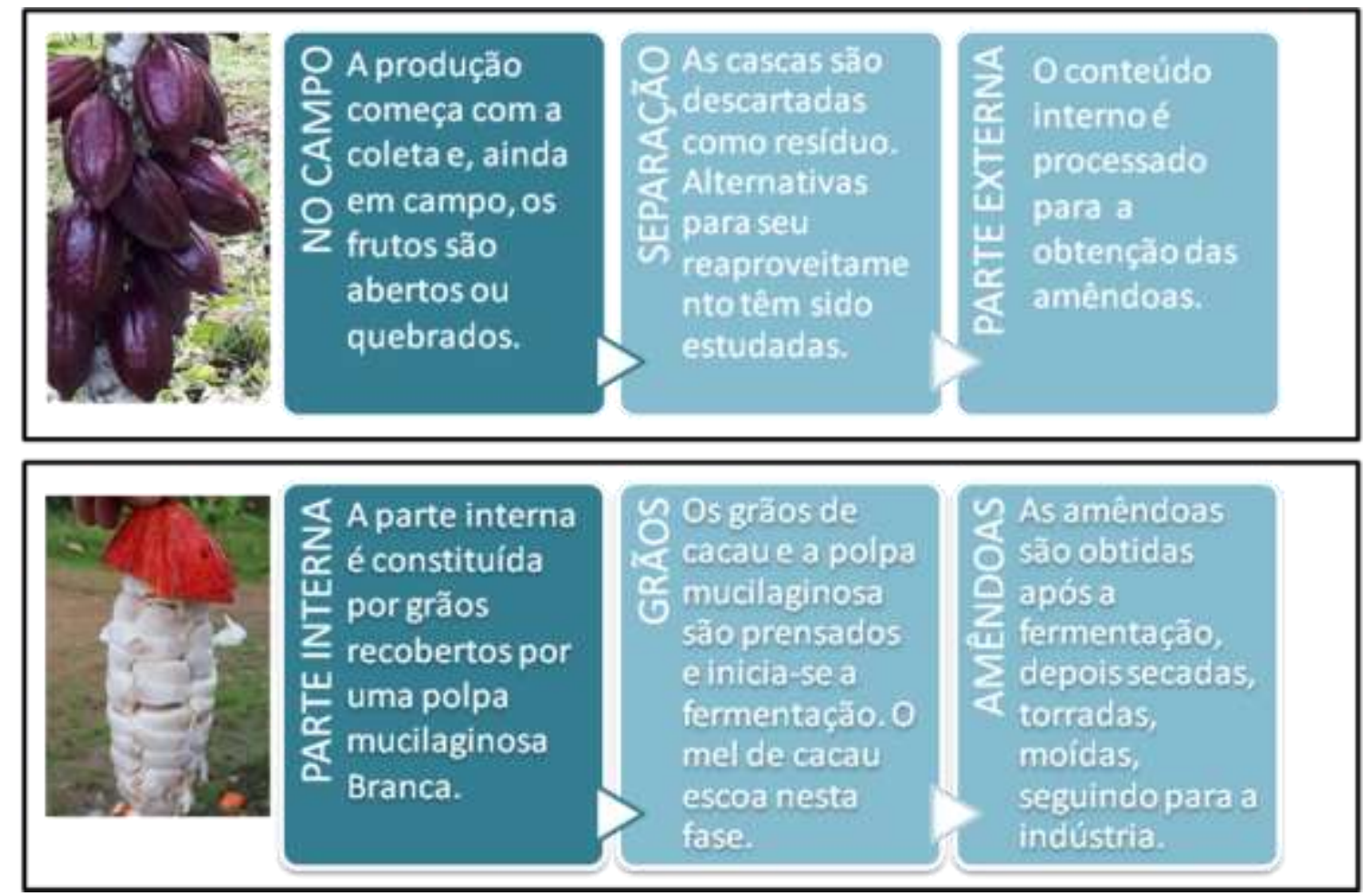

Fonte: Autores.

\section{Características Físico-Químicas, Propriedades Biológicas e Potencial Industrial do Mel de Cacau}

Alternativas para o aproveitamento e aumento do valor agregado do mel de cacau têm sido buscadas por produtores e pesquisadores. $\mathrm{O}$ aproveitamento deste subproduto agroindustrial é de grande importância para os países que são grandes produtores de cacau, o Brasil, onde a cultura do cacau ocupa uma área de 672.435 ha e gera uma média de 52.413 toneladas de resíduos/ano (Leite et al., 2019). A composição físico-química e diversas características do mel de cacau são descritas na literatura. Alguns parâmetros avaliados não apresentam diferenças significativas entre os trabalhos, como, por exemplo, a acidez titulável, a porcentagem de cinzas e o teor de ácidos não voláteis (Tabela 1). Em relação a este último parâmetro, os valores descritos para o mel de cacau não só coincidem entre si, como também, com o valor determinado para a polpa de cacau. Os valores encontrados para umidade variaram entre 74,94 e $87,22 \%$, o que está de acordo com a consistência aquosa do mel de cacau.

O teor de fibras no mel de cacau é baixo, mas ainda assim, os valores indicam que este alimento não é uma boa fonte de fibras, tendo em vista que o valor mínimo estipulado para um alimento a ser considerado fonte de fibras é de $2,5 \mathrm{~g}$ de fibra por porção (Brasil, 2012). Já a acidez natural do mel de cacau agrega valor ao mesmo, pois o meio ácido limita o crescimento de algumas espécies de microrganismos, tornando o meio restrito a bactérias láticas e acéticas, bolores e leveduras. 
Tabela 1 - Características físico-químicas do mel de cacau descritas na literatura.

\begin{tabular}{|c|c|c|c|c|c|}
\hline Análise & Origem & Produto & $\begin{array}{c}\text { Métodos de obtençãa e } \\
\text { processamento }\end{array}$ & Valor & Fonte \\
\hline \multirow{2}{*}{ Umidade $(\%)$} & Uruçuca, Bahia Brasil & MC & Esterilização/Congelamento & $87,22^{\mathrm{a}}$ & Melo Neto et al., 2016 \\
\hline & Sul da Bahia Brasil & MC & Congelamento & $83,21^{\mathrm{d}}$ & Santos et al., 2014 \\
\hline \multirow{2}{*}{ Cinzas $(\%)$} & Uruçuca, Bahia Brasil & $\mathrm{MC}$ & Esterilização/Congelamento & $0,23^{\mathrm{a}}$ & Melo Neto et al., 2016 \\
\hline & Sul da Bahia Brasil & MC & Congelamento & $0,26^{\mathrm{d}}$ & Santos et al., 2014 \\
\hline Fibras (\%) & Sudeste da Bahia, Brasil & $\mathrm{MC}$ & $\begin{array}{l}\text { Esterilização/Congelamento } \\
\text { Liofilização }\end{array}$ & $1,41^{\mathrm{b}}$ & Silva et al., 2014 \\
\hline \multirow{3}{*}{$\mathrm{pH}$} & Milagro, Guayas Equador & MC & $\begin{array}{l}\text { Fermentação/Filtração } \\
\text { Liofilização }\end{array}$ & $3,58^{\mathrm{i}}$ & Balladares, 2016 \\
\hline & Uruçuca, Bahia Brasil & MC & Esterilização/Congelamento & $2,76^{\mathrm{i}}$ & Melo Neto et al., 2016 \\
\hline & Sul da Bahia, Brasil & $\mathrm{MC}$ & Congelamento & $3,3^{\mathrm{j}}$ & Santos et al., 2014 \\
\hline \multirow{4}{*}{$\begin{array}{l}\text { Glicose } \quad \mathrm{e} \\
\text { frutose }(\%)\end{array}$} & Milagro, Guayas Equador & MC & $\begin{array}{l}\text { Fermentação/Filtração } \\
\text { Liofilização }\end{array}$ & $6,39^{\mathrm{k}}$ & Balladares, 2016 \\
\hline & Uruçuca, Bahia Brasil & $\mathrm{MC}$ & Esterilização/Congelamento & $10,2^{\mathrm{a}}$ & Melo Neto, 2016 \\
\hline & Sul da Bahia Brasil & $\mathrm{MC}$ & Prensagem in natura & $10-18^{\mathrm{n}}$ & Oetterer, 2006 \\
\hline & Uruçuca, Bahia Brasil & $\mathrm{PC}$ & Despolpa/Congelamento & 10 a $15^{1}$ & Passos et al., 1986 \\
\hline \multirow{3}{*}{ Sacarose (\%) } & Milagro, Guayas Equador & MC & $\begin{array}{l}\text { Fermentação/Filtração } \\
\text { Liofilização }\end{array}$ & $2,15^{\mathrm{k}}$ & Balladares, 2016 \\
\hline & Uruçuca, Bahia Brasil & MC & Esterilização/Congelamento & $4,06^{\mathrm{a}}$ & Melo Neto et al., 2016 \\
\hline & Sudeste da Bahia Brasil & $\mathrm{MC}$ & $\begin{array}{l}\text { Esterilização/Congelamento } \\
\text { Liofilização }\end{array}$ & $1,7^{\mathrm{e}, \mathrm{h}}$ & Silva et al., 2014 \\
\hline \multirow{2}{*}{$\begin{array}{l}\text { Sólidos } \\
\text { solúveis } \\
\text { totais }\left({ }^{\circ} \text { Brix }\right)\end{array}$} & Milagro, Guayas Equador & MC & $\begin{array}{l}\text { Fermentação/Filtração } \\
\text { Liofilização }\end{array}$ & $19,6^{\mathrm{c}}$ & Balladares et al., 2016 \\
\hline & Uruçuca, Bahia Brasil & MC & Prensagem in natura & $14,03^{\mathrm{m}}$ & Melo Neto et al., 2016 \\
\hline \multirow{3}{*}{$\begin{array}{l}\text { Vitamina C } \\
(\mathrm{mg} / 100 \\
\text { gramas) }\end{array}$} & Sudeste da Bahia Brasil & MC & $\begin{array}{l}\text { Esterilização/Congelamento } \\
\text { Liofilização }\end{array}$ & $10,9^{\mathrm{b}}$ & Silva et al., 2014 \\
\hline & Itabuna, Bahia Brasil & $\mathrm{PC}$ & Despolpa/Congelamento & $7,64^{\mathrm{c}}$ & Penha e da Matta, 1998 \\
\hline & Uruçuca, Bahia Brasil & $\mathrm{PC}$ & Despolpa/in natura & $10,0^{\mathrm{g}}$ & Passos et al., 1986 \\
\hline \multirow{2}{*}{ Pectina $(\%)$} & Sul da Bahia Brasil & $\mathrm{MC}$ & Prensagem/in natura & 0,9 a $2,5^{\mathrm{n}}$ & Oetterer, 1999 \\
\hline & Uruçuca, Bahia, Brasil & $\mathrm{PC}$ & Despolpa/in natura & 0,9 a $1,9^{f}$ & Passos et al., 1986 \\
\hline $\begin{array}{l}\text { Acidez } \\
\text { titulável (\%) }\end{array}$ & Uruçuca, Bahia Brasil & MC & Esterilização/Congelamento & $0,7^{\mathrm{a}}$ & Melo Neto et al., 2016 \\
\hline \multirow{2}{*}{$\begin{array}{l}\text { Ácidos não } \\
\text { voláteis (\% } \\
\text { ácido cítrico) }\end{array}$} & Sul da Bahia, Brasil & $\mathrm{MC}$ & Prensagem in natura & $\begin{array}{l}0,77^{\mathrm{a}} \\
1,50^{\mathrm{n}}\end{array}$ & Oetterer, 1999 \\
\hline & Uruçuca, Bahia Brasil & PC & Despolpa in natura & $\begin{array}{l}0,77^{\mathrm{a}} \\
1,52^{\mathrm{n}}\end{array}$ & Passos et al.,1986 \\
\hline
\end{tabular}

MC: mel de cacau, PC: Polpa de cacau. ${ }^{\mathrm{a}}$ Adolfo Lutz, 2008; ${ }^{\mathrm{b}} \mathrm{AOAC}, 2010 ;{ }^{\mathrm{c}} \mathrm{AOAC}, 2005 ;{ }^{\mathrm{d}}$ AOAC, 2002; ${ }^{\mathrm{e}}$ Matissek et al., $1998 ;{ }^{\mathrm{f}}$ Adomako, 1974; ${ }^{\mathrm{g}} \mathrm{Cox}$ e Person, 1962; hMiller, 1959; ${ }^{\mathrm{i}}$ Leitura direta em pHmetro; ${ }^{\mathrm{j}}$ Leitura direta em titulador; ${ }^{\mathrm{k}}$ Cromatografia líquida de alta eficiência

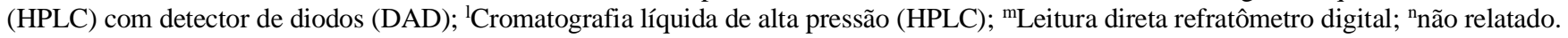
Fonte: Autores.

Os teores de sólidos solúveis totais descritos para o mel de cacau utilizando amostras de origem brasileira foram 11,5 (Santos et al., 2012) e 14,0 ${ }^{\circ}$ Brix (Melo Neto et al., 2016). Sendo um suco de sabor agridoce, é coerente a presença de glicose e frutose no mel de cacau, cujos teores variam de acordo com a região, ficando entre 8,6-18\% nas amostras brasileiras (Balladares et al., 2016). A presença de açúcares no mel de cacau confere propriedades nutricionais interessantes a este 
produto, colabora para a textura e pode ser um diferencial importante para a produção de bebidas e outros alimentos fermentados a partir do mel de cacau.

$\mathrm{O}$ uso de diferentes processos de coleta e estocagem dos frutos e do mel de cacau corrobora os resultados diferentes para alguns parâmetros apresentados na Tabela 1, como o teor de glicose e frutose. Balladares et al. (2016) por exemplo, coletaram o mel de cacau estudado durante três dias ao longo do experimento realizado em laboratório, armazenando-o em vidro e acondicionando-o a $4{ }^{\circ} \mathrm{C}$. Melo Neto et al. (2016) coletaram frutos sadios e maduros em seu trabalho e acondicionaram o mel de cacau obtido em garrafas esterilizadas, congeladas a $-18{ }^{\circ} \mathrm{C}$. Silva et al. (2014) obtiveram mel de cacau em laboratório a partir de frutos maduros colhidos aleatoriamente em três propriedades distintas, enquanto Passos et al. (1986) apresentaram valores de sacarose para polpa de cacau ainda não degradada em mel de cacau. Estes fatores também poderiam justificar os diferentes valores encontrados para o teor de sólidos solúveis (brix) e sacarose relatados.

Os açúcares detectados no mel de cacau são os mesmos açúcares encontrados em frutas, cana de açúcar e beterraba. Estes carboidratos, também conhecidos como adoçantes nutritivos, são a principal fonte de energia para o corpo humano, fornecendo em média quatro calorias por grama. O mel de cacau apresenta pequena quantidade de vitamina C. Ainda assim, considerando-se a ingestão diária recomendada (IDR) de vitamina $\mathrm{C}$ de $45 \mathrm{mg}$ para adultos, o mel de cacau pode contribuir com cerca de $24 \%$ da IDR de vitamina C, com a ingestão de apenas $100 \mathrm{~mL}$ por dia.

Por si só, o mel de cacau apresenta diversas características que permitem visualizar seu aproveitamento em uma série de produtos alimentícios, a começar pela ampliação do seu uso como suco. Seu sabor doce permite considerar aplicações como fonte de doçura em produtos diversos, em substituição ao açúcar de cana. Associados ao sabor doce, a acidez moderada e o teor de vitamina $\mathrm{C}$ tornam o mel de cacau um interessante ingrediente para o preparo de sucos mistos de frutas, uma tendência que vem crescendo muito na atualidade. Porém, uma importante característica do mel de cacau é sua semelhança, em termos de sabor, com a polpa de cacau. A Tabela 2 apresenta uma comparação entre mel, polpa e suco de polpa de cacau, onde podem ser observadas semelhanças também em diversas propriedades físico-químicas e conteúdo de macro e micronutrientes.

Além dos atributos físico-químicos do mel de cacau já discutidos, a Tabela 2 mostra ainda que o mel de cacau apresenta baixa porcentagem de gordura e possui micronutrientes minerais importantes como cálcio e potássio, sendo que o teor de potássio no mel de cacau supera os valores relatados para a polpa e o suco. 
Tabela 2 - Comparação de dados físico-químicos e teor de macro e micronutrientes da polpa de cacau, mel de cacau e suco de polpa de cacau.

\begin{tabular}{llll}
\hline Parâmetros & Polpa de cacau & Mel de cacau & Suco da polpa de cacau \\
\hline Físico-químicos & & & \\
\hline Sólidos solúveis totais (brix) & $16^{\mathrm{d}}$ & $14,3^{\mathrm{c}}$ & $16,7^{\mathrm{a}}$ \\
Acidez (\% ácido cítrico) & $0,9^{\mathrm{d}}$ & $0,7^{\mathrm{c}}$ & $1,0^{\mathrm{a}}$ \\
Densidade $(\mathrm{g} / \mathrm{mL})$ & $1,0^{\mathrm{d}}$ & $1,1^{\mathrm{b}}$ & $1,1^{\mathrm{d}}$ \\
$\mathrm{pH}$ & $3,8^{\mathrm{d}}$ & $3,6^{\mathrm{b}}$ & $3,8^{\mathrm{d}}$ \\
\hline Macronutrientes & & & \\
\hline Fibras totais $(\%)$ & $1,1^{\mathrm{a}}$ & $1,4^{\mathrm{f}}$ & $1,2^{\mathrm{a}}$ \\
Sacarose $(\mathrm{g} / 100 \mathrm{~g})$ & $8,2^{\mathrm{h}}$ & $2,2^{\mathrm{b}}$ & $4,1^{\mathrm{g}}$ \\
Frutose $(\mathrm{g} / 100 \mathrm{~g})$ & $4,4^{\mathrm{h}}$ & $4,4^{\mathrm{b}}$ & $1,0^{\mathrm{g}}$ \\
Glicose $(\mathrm{g} / 100 \mathrm{~g})$ & $3,7^{\mathrm{h}}$ & $2,1^{\mathrm{b}}$ & $2,3^{\mathrm{g}}$ \\
Gorduras $(\%)$ & $0,2^{\mathrm{a}}$ & $0,3^{\mathrm{e}}$ & $0,2^{\mathrm{a}}$ \\
Proteína $(\%)$ & $0,9^{\mathrm{d}}$ & $1,1^{\mathrm{e}}$ & $0,6^{\mathrm{a}}$ \\
Cinzas $\left(\mathrm{g} / 100 \mathrm{~g}{ }^{-1}\right)$ & $0,5^{\mathrm{a}}$ & $0,3^{\mathrm{e}}$ & $0,5^{\mathrm{a}}$ \\
\hline Micronutrientes minerais & & & \\
\hline Minerais $\left(\mathrm{mg} / 100 \mathrm{~mL}^{-1}\right)$ & $0,3^{\mathrm{i}}$ & $0,1^{\mathrm{h}}$ & $0,2^{\mathrm{a}}$ \\
Cálcio $(\mathrm{mg} / \mathrm{kg})$ & $54^{\mathrm{a}}$ & $171,5^{\mathrm{h}}$ & $73^{\mathrm{a}}$ \\
Fosforo $(\mathrm{mg} / \mathrm{kg})$ & $140^{\mathrm{a}}$ & $62,5^{\mathrm{h}}$ & $123^{\mathrm{a}}$ \\
Potássio $(\mathrm{mg} / \mathrm{kg})$ & $1700^{\mathrm{a}}$ & $950^{\mathrm{h}}$ & $2020^{\mathrm{a}}$ \\
Magnésio $(\mathrm{mg} / \mathrm{kg})$ & $200^{\mathrm{a}}$ & $82,5^{\mathrm{h}}$ & $220^{\mathrm{a}}$ \\
Sódio $(\mathrm{mg} / \mathrm{kg})$ & $6^{\mathrm{a}}$ & $30,5^{\mathrm{h}}$ & $11^{\mathrm{a}}$ \\
\hline
\end{tabular}

${ }^{\mathrm{a}}$ EFSA, 2019; ${ }^{\mathrm{b}}$ Balladares, 2016; ${ }^{\mathrm{c}}$ Melo Neto et al., 2016; ${ }^{\mathrm{d}}$ Torres et al., 2016; ${ }^{\mathrm{e}}$ Santos et al., 2014; ${ }^{\mathrm{f}}$ Silva et al., 2014; ${ }^{\mathrm{g}}$ Oddoye et al., 2013; hPenha e da Matta, 1998; 'Passos et al.,1986. Fonte: Autores.

Outro importante atributo do mel de cacau no que diz respeito à sua incorporação em novos produtos é a presença de pectina, entre $0,8 \%$ (Santos, 2012) e 2,5\% (Oetterer, 2006) valor comparável ao conteúdo de pectina determinado para a polpa de cacau (0,9-1,9) (Passos et al., 1986). A pectina tem importante papel em projetos de alimentos pela capacidade de modular a resposta de humanos aos efeitos inibitórios na ingestão e absorção de lipídeos na alimentação (Zhou et al., 2021). O desenvolvimento de novas aplicações para o mercado de pectina, atualmente abastecido principalmente por extração deste composto a partir de cascas de laranja e de bagaço de maçã, tem sido focado no design de processos extrativos mais eficientes e mais competitivos. Diversos métodos extrativos não convencionais, com credencial verde, assistidos por micro-ondas, ultrassom e enzimas têm sido desenvolvidos visando aumentar de forma sustentável, o rol de frutas utilizadas como fonte de pectina (Picot-Allain et al., 2020). Cascas de laranja possuem alto teor de pectina, podendo chegar a 5,5\%, mas outras fontes como bagaço de maçã $(1,5-2,5 \%)$, banana $(0,7-1,2 \%)$ e mamão $(0,7-1,0 \%)$ possuem níveis comparáveis aos descritos para o mel de cacau (Canteri et al., 2012). Algumas aplicações industriais interessantes da pectina são apresentadas na Tabela 3. 
Tabela 3 - Matérias primas fontes de pectina e respectivas aplicações industriais.

\begin{tabular}{lll}
\hline Matéria-prima & Aplicação & Fonte \\
\hline Mel de cacau & Geleia sem adição de açúcar & Santos, 2012 \\
Beterraba & Texturizante para produtos com baixo teor de açúcar & Lara-Espinoza et al., 2021 \\
Casca de caqui & Emulsificante & Jiang et al., 2020 \\
Casca de citrus & Bioplástico & Mellinas et al., 2020 \\
Cascas de jaca & Geleificante, estabilizante, emulsificante e texturizante & Naik et al., 2020 \\
Casca de laranja & Filmes comestíveis & Jridi et al., 2020 \\
Casca de manga & Embutidos secos & Wongkaew et al., 2020 \\
Cascas de maracujá & Modificador reológico em produtos com baixo teor de açúcar & Abboud et al., 2020 \\
Casca de tomate & Inibidor de corrosão em metais & Halambek et al., 2020 \\
Cera de carnaúba e & Bionanocompósitos para filmes e coberturas comestíveis & Costa e Silva et al., 2019 \\
óleo de neem & (novas embalagens) & \\
\hline
\end{tabular}

Fonte: Autores.

O cacau é rico em compostos bioativos como polifenois, cujo teor, assim como sua capacidade funcional, características sensoriais e qualidade final do chocolate e produtos derivados podem variar dependendo do grau de maturação do cacau, genótipo, alterações durante a pós-colheita, condições dos processos tecnológicos e armazenamento (Febrianto \& Zhu, 2019). O aumento do consumo de chocolate e derivados de cacau está relacionado, entre outros fatores, aos benefícios destes para a saúde, muitos dos quais relacionados à presença de altos teores de antioxidantes. Diversos estudos sugerem que haja uma estreita relação entre o consumo de cacau e benefícios cardiovasculares. O consumo de cacau também gera efeitos neuroprotetores e anti-inflamatórios, inclusive associados a doença renal crônica, mas tais benefícios estão ligados à porcentagem de cacau, quando este é consumido na forma de chocolate (Fanton et al., 2021). A presença de compostos fenólicos e atividade antioxidante no mel de cacau foi relatada por Ramos et al. (2013).

Um dos entraves a serem superados para ampliar o leque de aplicações industriais do mel de cacau é a vida de prateleira curta. Esta característica é decorrente da alta carga microbiana presente no fruto do cacau, associada às condições artesanais do processo de pós-colheita e potencializada por materiais impróprios, utilizados na construção de cochos de fermentação e de utensílios. Desta forma, mesmo após o mel de cacau ser retirado do cocho de fermentação, os microrganismos continuam em atividade, provocando alterações reológicas e sensoriais neste insumo. Assim, um grande obstáculo encontrado na utilização do mel de cacau é uma conservação adequada, capaz de evitar degradação em função do processo fermentativo contínuo (Silva et al., 2014).

A pasteurização é um processo bem conhecido e que poderia vir a ser uma opção para a conservação do mel de cacau. Tratamentos térmicos moderados utilizando uma combinação de temperatura e duração para a inativação de microrganismos já são aplicados na produção de sucos e xaropes industrializados (Ishara, \& Gunasena 2021). Resultados bem-sucedidos empregando-se metabissulfito de potássio $(32 \mathrm{~g} / 100 \mathrm{~mL})$ para o preparo de geleia de mel de cacau foi relatado $($ Oetterer, 1999). Além do metabissulfito, aditivos como ácidos sórbicos (sorbatos) e benzoatos, classificados como conservadores de alimentos, também têm sido adicionados a sucos de frutas para aumentar sua vida útil. Santos (2012) descreveu o desenvolvimento de formulações de geleias utilizando sorbato como conservante. Processos alternativos, tais como radiação 
gama e micro-ondas, poderão ser adotados no futuro visando minimizar efeitos negativos de processos de conservação convencionais nos constituintes termolábeis do mel de cacau como antioxidantes e vitamina C (Teixeira \& Monteiro, 2006).

\section{Potencial Tecnológico do Mel de Cacau na Indústria de Alimentos}

O sabor característico do mel de cacau abrange diversas possibilidades para sua utilização e aplicação na indústria de alimentos. Produtos como bebidas alcóolicas, xaropes, geleias e licores, elaborados com o mel de cacau já têm sido produzidos por comunidades extrativistas, cooperativas e produtores artesanais de alimentos. Alguns trabalhos relatando produtos contendo mel de cacau são apresentados na Tabela 4.

Tabela 4 - Alguns alimentos preparados com mel de cacau descritos na literatura.

\begin{tabular}{|c|c|c|c|}
\hline Origem do mel de cacau & Processamento & Produto & Fonte \\
\hline $\begin{array}{l}\text { Mel de cacau pasteurizado } \\
\text { ou não/ Nicarágua }\end{array}$ & Fermentação & Álcool e vinho & Corrales et al., 2015 \\
\hline $\begin{array}{c}\text { Mel de cacau } \\
\text { pasteurizado/Equador }\end{array}$ & $\begin{array}{l}\text { Adição de água, carboidratos, sais } \\
\text { minerais, conservante (benzoato de } \\
\text { sódio) e ácido cítrico }\end{array}$ & Bebida hidratante & Santana et al., 2018 \\
\hline $\begin{array}{c}\text { Mel de cacau } \\
\text { pasteurizado/Brasil }\end{array}$ & $\begin{array}{c}\text { Adição de grãos de kefir e solução } \\
\text { açucarada), inoculação e fermentação } \\
\left(10-25^{\circ} \mathrm{C}\right)\end{array}$ & Bebida de Kefir & Puerari et al., 2012 \\
\hline Mel de cacau/Brasil & $\begin{array}{c}\text { Adição de água de coco e açúcar e } \\
\text { pasteurização }\end{array}$ & $\begin{array}{l}\text { Bebida mista (mel de } \\
\text { cacau e água de coco) }\end{array}$ & Feitosa et al., 2014 \\
\hline Mel de cacau/Equador & $\begin{array}{l}\text { Estabilização com meta bisulfito de } \\
\text { sódio e ácido ascórbico; adição de } \\
\text { açúcar e pectina. }\end{array}$ & $\begin{array}{l}\text { Geleia da mucilagem } \\
\text { fermentada de cacau }\end{array}$ & Torres et al., 2015 \\
\hline Mel de cacau/Brasil & $\begin{array}{l}\text { Adição de açaí, sacarose e pectina } \\
\text { cítrica, concentração a } 94^{\circ} \mathrm{C}\end{array}$ & $\begin{array}{l}\text { Geleia mista de açaí e } \\
\text { mel de cacau }\end{array}$ & $\begin{array}{l}\text { Melo Neto et al., } \\
2013\end{array}$ \\
\hline Mel de cacau/Brasil & $\begin{array}{l}\text { Adição de adoçante e pectina e } \\
\text { concentração por meio de cocção }\end{array}$ & $\begin{array}{l}\text { Geleia sem adição de } \\
\text { açúcar }\end{array}$ & Santos et al., 2014 \\
\hline Mel de cacau/Equador & $\begin{array}{l}\text { Adição de metabisulfito de sódio e } \\
\text { fermentação por } S \text {. cerevisiae }\end{array}$ & $\begin{array}{l}\text { Vinagre de mel de } \\
\quad \text { cacau }\end{array}$ & $\begin{array}{l}\text { Garcia e Moreta, } \\
2013\end{array}$ \\
\hline
\end{tabular}

Fonte: Autores.

Ferreira e Dias (1982) analisaram a produção de mel de cacau como uma atividade econômica e concluíram que apenas $0,4 \%$ da produção deste subproduto estava sendo usada. $\mathrm{O}$ aproveitamento deste subproduto não aumentou significativamente desde então, devido à escassez de estudos sobre a conservação e melhoria tecnológica e às características físico-químicas, bioquímicas e microbiológicas do mel de cacau, dificultando sua exploração e comercialização (Silva et al., 2014). Mas já existem iniciativas como o envase e congelamento do mel de cacau in natura e o uso na produção de alimentos e bebidas tem aumentado (Santos \& Kalid, 2020).

\section{Considerações Finais}

O mel de cacau é um subproduto estratégico no atual cenário de ampliação do mercado de cacau e derivados e do aumento do consumo de chocolate. Diversos atributos nutricionais e sensoriais importantes tornam o mel de cacau um ótimo 
ingrediente para a indústria de alimentos e como ingrediente para a crescente indústria de gastronomia. Além de sua comercialização como suco natural, o mel de cacau pode ser incorporado em bebidas mistas e na produção de alimentos como edulcorante, saborizante e mesmo como ingrediente funcional. Diversos obstáculos tecnológicos ainda precisam ser superados para o aproveitamento total do mel de cacau, como o aumento do rendimento, os problemas relacionados à curta vida de prateleira do produto in natura, às contaminações, dentre outros. Por outro lado, a incorporação do mel de cacau na cadeia produtiva aumenta a percepção dos consumidores quanto à preocupação com a sustentabilidade da cadeia produtiva do cacau e derivados, colaborando também para a bioeconomia local e mesmo nacional. Aos poucos, o mel de cacau tem se tornado conhecido nos países produtores, principalmente em feiras, mas também por turistas que participam de degustações em fazendas de cacau. A incorporação do mel de cacau em chocolates com apelo regional, seu uso na produção de geleias e bebidas fermentadas também colaboram para um cenário de aproveitamento total do mel de cacau no futuro próximo.

\section{Agradecimentos}

Os autores agradecem o apoio financeiro da Fundação de Amparo à Pesquisa do Estado de Minas Gerais (FAPEMIG PPM-00255-18), Conselho Nacional de Desenvolvimento Científico e Tecnológico (CNPq 304922/2018-8 e 141601/2018 -3), Coordenação de Aperfeiçoamento de Pessoal de Nível Superior (CAPES, código 001) e Instituto Nacional de Ciência e Tecnologia - INCT BioNat, concessão nº 465637/2014-0, Brasil.

\section{Referências}

Abboud, K. Y., Iacomini, M., Simas, F. F., \& Cordeiro, L. M. (2020). High methoxyl pectin from the soluble dietary fiber of passion fruit peel forms weak gel without the requirement of sugar addition. Carbohydrate Polymers, 246, 116616.

Adomako, D. (1974). Chemical characterization of cocoa pectin. Chemistry and Industry, 21, 873-74.

ABICAB - Associação Brasileira de Industriais de Chocolates, Amendoim e Balas. (2020). Produtores de cacau ABICAB (2020) produção nacional de chocolate cresce no terceiro trimestre. http://www.abicab.org.br/noticias/producao-nacional-de-chocolate-cresce-no-terceiro-trimestre/

AOAC - Association of Official Analytical Chemistry. (2010). Official methods of analysis of the association of official analytical chemists, (18a ed.). Gaithersburg, MD: AOAC International.

AOAC - Association of Official Analytical Chemists. (2005). Official methods of the association of official analytical chemistry, (16a ed.). Washington.

AOAC - Association of Official Analytical Chemists. (2002). Official methods of analysis of AOAC International, 2, (17a ed.). Washington.

Balladares, C. G., Chóez-Guaranda, J. P. I., Gonzlez, S., Sosa, J., Viteri, D., Barragn, R., Quijano, A., Isabel, M. P., \& Santana, M. (2016). Physicochemical characterization of Theobroma cacao L. mucilage, in Ecuadorian coast. Emirates Journal of Food and Agriculture, p. 741-45.

Agência Nacional de Vigilância Sanitária. Resolução - RDC No 54, de 12 de novembro de 2012. Dispõe sobre o Regulamento Técnico sobre Informação Nutricional Complementar. Diário Oficial da União.

Agência Nacional de Vigilância Sanitária. Resolução-RDC $N^{o} 269$, de 22 de setembro de 2005. Dispõe regulamento técnico sobre ingestão diária recomendada (IDR) para proteína, vitaminas e minerais. Diário Oficial da União.

Ministério da Indústria, Comércio Exterior e Serviços. Estudo de Competitividade do Cacau e Chocolate no Brasil: Desafios na Produção e Comércio Global. Brasília.

BUSINESSWIRE. (2020). Chocolate market in europe analysis highlights the impact of COVID-19 (2020-2024). Demand for Seasonal Chocolates to boost the Market Growth, Technavio. https://www.businesswire.com/news/home/20200709005654/en/Chocolate-Market-Europe-Analysis-Highlights-Impact-COVID19.

Canteri, M. H. G., Moreno, L., Wosiacki, G., \& Scheer, A de P. (2012). Pectina: da matéria-prima ao produto final. Polímeros, 22 (2), $149-57$.

Castro e Silva, P., Oliveira, A. C. S., Pereira, L. A. S., Valquíria, M., Carvalho, G. R., Miranda, K. W. E. \& Oliveira, J. E. (2019). Development of bionanocomposites of pectin and nanoemulsions of carnauba wax and neem oil pectin/carnauba wax/neem oil composites. Polymer Composites, 41 (3), 85870 .

CEPLAC - Centro de Pesquisa do Cacau. (2019). Brasil quer ganhar posições na produção mundial de cacau e chocolate. http://www.agricultura.gov.br/noticias/brasil-quer-retomar-protagonismo-no-cenario-global-de-cacau-e-chocolate. 
CBI - Centre for The Promotion of imports from developing countries. (2020). What is the demand for cocoa on the European market? https://www.cbi.eu/market-information/cocoa/trade-statistics.

Corrales, A. V. D., Leiva, Y. M. C., \& Lazo, Z. M. M. (2015). Evaluación y determinación de la producción de vino y alcohol a partir de exudado de cacao, cooperativa multifuncional cacaotera la campesina R.L, del municipio de Matiguás en el departamento de Matagalpa. A Revista Científica Hel Higo, 5 (1), 216.

Cox, H. E., \& Person, D. (1962). The chemical analysis of foods. Chemical Publishing.

Euromonitor. (2016). Global trends and developments in Cocoa ingredients (world). https://www.euromonitor.com/global-trends-and-developments-in-cocoaingredients-world/report

EFSA - European Food Safety Authority. (2019). Technical report on the notification of pulp from Theobroma cacao L. as a traditional food from a third country pursuant to Article 14 of Regulation (EU) 2015/2283. EFSA Supporting Publications, 16 (11), 1724E.

Fanton, S., Cardozo, L. F. M. F., Combet, E., Shiels, P. G., Stenvinkel, P., Vieira, I. O., Narciso, H. R., Schmitz, J., \& Mafra D. (2021). The sweet side of dark chocolate for chronic kidney disease patients. Clinical Nutrition, 40 (1), 15-26.

Febrianto, N. A., \& Zhu, F. (2019). Intravariety diversity of bioactive compounds in trinitario cocoa beans with different degrees of fermentation. Journal of Agricultural and Food Chemistry, 67 (11), 3150-58.

Feitosa, A., Andrade, R. O., Costa, D. P., Batista, D. V. S., \& Cardoso, R. L. (2014). Desenvolvimento, caracterização físico-química e sensorial de bebida mista de mel de cacau com água de coco. Enciclopédia Biosfera, 10 (18), 1183-92.

Ferreira, H. I. S., \& Dias, S. D. P. (1982). Perfil econômico e social da produção de mel de cacau. Boletim Técnico-Comissão Executiva do Plano de Recuperação Econômico-Rural da Lavoura Cacaueira, 97, 38.

Freire, E. S., Romeu, A. P., Passos, F. V., Passos, F. L., Mororó, R. C., Schawan, R. F., Llamosas, A., Chepote, R. E., Santana, M. B. M., \& Ferreira, H. I. S. (1990). Aproveitamento de resíduos e subprodutos da pós colheita do cacau. Centro de Pesquisas do Cacau (CEPEC) - CEPLAC. $53^{\circ}$ reunião da Aliança dos Países Produtores de Cacau. Ilhéus, BA, Brasil.

García, S. V., \& Moreta, F. A. (2013). Optimización y aprovechamiento del residuo (exudado del mucílago) de la almendra fresca del cacao (Theobroma cacao L.) Ccn51 en la elaboración de vinagre. Revista de Investigação Científica, 4 (4), 7-19.

Halambek, J., Cindrić, I., \& Grassino, A. N. (2020). Evaluation of pectin isolated from tomato peel waste as natural tin corrosion inhibitor in sodium chloride/acetic acid solution. Carbohydrate Polymers, 234, 115940.

ICCO - Organização Internacional do Cacau. (2020). Quarterly bulletin of cocoa statistics, year 2019/2020. https://www.icco.org/wpcontent/uploads/Production-QBCS-XLVI-No-4.pdf

Ishara, A. W. S., \& Gunasena, G. D. D. K. (2021). Heat resistant moulds in pasteurized fruit syrups. European Journal of Agriculture and Food Sciences, 3 $(1), 104-11$

Instituto Adolfo Lutz. (2008). Normas Analíticas do Instituto Adolfo Lutz: Métodos físicos e químicos para análises de alimentos: Instituto Adolfo Lutz, pp 1020 .

Jiang, Y., Xu, Y., Li, F., Li, D., \& Huang, Q. (2020). Pectin extracted from persimmon peel: A physicochemical characterization and emulsifying properties evaluation. Food Hydrocolloids, 101, 105561.

Jridi, M., Abdelhedi, O., Salem, A., Kechaou, H., Nasri, M., \& Menchari, Y. (2020). Physicochemical, antioxidant and antibacterial properties of fish gelatinbased edible films enriched with orange peel pectin: Wrapping application. Food Hydrocolloids, 103, 105688.

Lara-Espinoza, C., Sanchez-Villegas, J. A., Lopez-Franco, Y., Carvajal-Millan, E., Troncoso-Rojas, R., Carvallo-Ruiz, T., \& Rascon-Chu, A. (2021). Composition, physicochemical features, and covalent gelling properties of ferulated pectin extracted from three sugar beet (Beta vulgaris L.) cultivars grown under desertic conditions. Agronomy, 11 (40), 2-13.

Lima, L. J., Almeida, M. H., Nout, M. R., \& Zwietering, M. H. (2011). Theobroma cacao L. "The food of the Gods": quality determinants of commercial cocoa beans, with particular reference to the impact of fermentation. Critical reviews in food science and nutrition, 51 (8), 731-61.

Leite, P. B., Machado, W. M., Guimarães, A. G., Carvalho, G. B. M., Teixeira, M. G. K., \& Druzian, J. I. (2019). Cocoa residual honey: Physicochemical characterization and potential as a fermentative substrate by Sacchromyces cerevisiae AWRI726. The Scientific World Journal, 1-7.

Matissek, R., Steiner, G., \& Schnepel, F. M. (1998). Análisis de los alimentos: fundamentos, métodos, aplicaciones. Acribia.

Mellinas C., Ramos M., Jiménez A., \& Garrigós M. C. (2020). Recent trends in the use of pectin from agro-waste residues as a natural-based biopolymer for food packaging applications. Materials, 13 (3), 673.

Melo Neto, B. A. D., Bonomo, R. C. F., Fontan, R. D. C. I., Ferreira, A. C. R., Gonçalves, G. R. F., \& Mello, D. L. N. (2016). Physicalchemical characterization and thermophysical properties of cocoa honey. Revista GEINTEC- Gestão, Inovação e Tecnologias, 6 (1), $2944-53$.

Melo Neto, B. A., Carvalho, E. A., Pontes, K. V., Barretto, W. D. S., \& Sacramento, C. K. D. (2013). Chemical, physico-chemical and sensory characterization of mixed açai (Euterpe oleracea) and cocoa's honey (Theobroma cacao) jellies. Revista Brasileira de Fruticultura, 35 (2), 587-93.

Mercado do Cacau. (2020). Mais de $90 \%$ dos brasileiros compraram chocolate para consumo dentro de casa em 2020. http://mercadodocacau.com/artigo/mais-de-90-dos-brasileiros-compraram-chocolate-para-consumo-dentro-de-casa-em-2020. 
Miller, G. L. (1959). Use of dinitrosalicylic acid reagent for determination of reducing sugar. Analytical Chemistry, 31(3), 426-28.

Naik, M., Rawson, A., \& Rangarajan, J. M. (2020). Radio frequency-assisted extraction of pectin from jackfruit (Artocarpus heterophyllus) peel and its characterization. Journal of Food Process Engineering, 43 (6), e13389.

Oetterer, M. (2006). Tecnologias de obtenção do cacau, produtos do cacau e do chocolate. In: Oetterer, M., Regitano d’Arce, M. A., \& Spoto, M. H. F. (Org.). Fundamentos de Ciência e Tecnologia de Alimentos. Manole, 1, 1-50.

Oddoye, E. O. K., Agyente-Badu, C. K., \& Gyedu-Akoto, E. (2012). Cocoa and its by-products: identification and utilization. Chocolate in Health and Nutrition, 23-37.

Passos, F. J. V., Pereira, V. P., Passos, F. M. L., \& Romeu, A. P. (1986). Obtenção e perspectivas do processamento térmico do suco e néctar da polpa do cacau. Boletim Técnico - Comissão Executiva do Plano da Lavoura Cacaueira (Brasil), 137, 30.

Penha, E. M., \& Matta, V. M. (1998). Características físico-químicas e microbiológicas da polpa de cacau, Pesquisa Agropecuária Brasileira - PAB, 33 (11), $1945-49$.

Pereira, A. S., Shitsuka, D. M., Pereira, F. J., \& Shitsuka, R. (2018). Metodologia da pesquisa científica. UFSM. https://repositorio.ufsm.br/bitstream/h andle/1/15824/Lic_Compu tacao_Metodologia-Pesquisa-Cientifica.pdf?sequence=1.

Picot-Allain, M. C. N., Ramasawmy, B., \& Emmambux, M. N. (2020). Extraction, characterisation, and application of pectin from tropical and sub-tropical fruits: A Review. Food Reviews International, 1-31.

Puerari, C., Magalhães, K. T., \& Schwan, R. F. (2012). New cocoa pulp-based kefir beverages: microbiological, chemical composition and sensory analysis. Food Research International, 48, 634-40.

Ramos, D. C., Silva, E. N., Su, F., Silva, M. V., \& Lannes, S. C. S. (2013). Composições alimentícias de chocolate e de gelado comestível contendo mel e cacau. Rio de Janeiro: República Federativa do Brasil - Ministério do Desenvolvimento, Indústria e do Comércio Exterior - Instituto Nacional da Propriedade Industrial.

Ruesgas-Ramón, M., Suárez-Quiroz, M. L., González-Ríos, O., Baréa, B., Cazals, G., Figueroa-Espinoza, M. C., \& Durand, E. (2020) Biomolecules extraction from coffee and cocoa by-and co-products using deep eutectic solvents. Journal of the Science of Food and Agriculture, 100 (1), 81-91.

Santana, P., Vera, J., Vallejo, C., \& Alvarez, A. (2018). Mucílago de cacao, nacional y trinitario para la obtención de una bebida hidratante. Universidad, Ciencia y Tecnología, 4, $179-89$.

Santos, C. O. (2012). Aproveitamento industrial de mel de cacau (Theobroma cacao L) na produção de geleia sem adição de açúcar. (Dissertação Mestrado em Ciência de Alimentos). Universidade Federal da Bahia. Salvador, BA, Brasil.

Santos, C. O. D., Bispo, E. D. S., Santana, L. R. R. D., \& Carvalho, R. D. S. D. (2014). Use of "cocoa honey" (Theobroma cacao L) for diet jelly preparation: an alternative technology. Revista Brasileira de Fruticultura, 36 (3), 2944-53.

Santos, F. C. G., \& Kalid, R. A. (2020). Prospecção tecnológica: um estudo de tecnologias aplicadas ao processamento e derivados de cacau. Pesquisa, Sociedade e Desenvolvimento, 9 (3), 56932354.

Silva, E. N., Ramos, D. C., Menezes, L. M., Souza, S. O., Lannes, S. C. S., \& Silva, M. V. (2014). Valor nutricional e capacidade antioxidante do mel de cacau (Theobroma cacao L.). Food Science Technology, 34 (4), 755-59.

Teixeira, M., \& Monteiro, M. (2006). Degradação da vitamina C em suco de fruta. Alimentos e Nutrição, 17 (2), $219-27$.

Torres, C. A. V., Ocampo, R. D., Rodríguez, W. M, Velasco, R. S., Chang, J. F. V., \& Cedeño, C. B. (2016). Utilización del mucílago de cacao, tipo nacional y trinitario, en la obtención de jalea. ESPAM Ciencia, 7 (1), 51-58.

Wongkaew, M., Sommano, S. R., Tangpao, T., Rachtanapun, P. E., \& Jantanasakulwong, K. (2020). Mango peel pectin by microwave-assisted extraction and its use as fat replacement in dried Chinese sausage. Foods, 9 (4), 450.

Zhou, M., Bi, J., Chen, J., Wang, R., \& Richel, A. (2021). Impacto das características da pectina na digestão de lipídios em condições gastrointestinais simuladas: Comparação de pectinas solúveis em água extraídas de diferentes fontes. Food Hydrocolloids, $112,106350$. 